\title{
METRIC SPACES WITH UNIQUE TANGENTS
}

\author{
Enrico Le Donne \\ ETH Zürich, Departement Mathematik \\ Rämistrasse 101, 8092 Zürich, Switzerland; enrico.ledonne@math.ethz.ch
}

\begin{abstract}
We are interested in studying doubling metric spaces with the property that at some of the points the metric tangent is unique. In such a setting, Finsler-Carnot-Carathéodory geometries and Carnot groups appear as models for the tangents.

The results are based on an analogue for metric spaces of Preiss's phenomenon: tangents of tangents are tangents. In fact, we show that, if $X$ is a general metric space supporting a doubling measure $\mu$, then, for $\mu$-almost every $x \in X$, whenever a pointed metric space $(Y, y)$ appears as a Gromov-Hausdorff tangent of $X$ at $x$, then, for any $y^{\prime} \in Y$, also the space $\left(Y, y^{\prime}\right)$ appears as a Gromov-Hausdorff tangent of $X$ at the same point $x$. As a consequence, uniqueness of tangents implies their homogeneity. The deep work of Gleason-Montgomery-Zippin and Berestovskiı̆ leads to a Lie group homogeneous structure on these tangents and a characterization of their distances.
\end{abstract}

\section{Introduction}

This paper shows that there is a relation between isometrically homogeneous spaces and uniqueness of tangents for metric spaces. It is a consequence of the work of Gleason, Montgomery-Zippin, Berestovskiı̌, Mitchell, and Margulis-Mostow that a finite-dimensional geodesic metric space with transitive isometry group has the property that at every point the tangent metric space is unique. Such a tangent is in fact a Carnot group equipped with a Carnot-Carathéodory distance. In the following paper we consider doubling-measured metric spaces with the property that at almost every point the tangent metric space is unique and show that almost all tangents have transitive isometry group. Consequently, if in addition the metric space is geodesic, then the tangents are almost surely Carnot groups equipped with Carnot-Carathéodory distances.

Our results are founded on the translation in the context of metric spaces of a fact that is well known in Geometric Measure Theory: tangent measures of tangent measures are tangent measures. In fact, let $\mu$ be a doubling measure in the Euclidean space $\mathbf{R}^{n}$. Then one can define the tangent measures of $\mu$ at a point $x \in \mathbf{R}^{n}$ by taking weak* limits of measures translated by $x$ and dilated by larger and larger factors. Namely, setting $T_{x, \rho}(y):=\rho(y-x)$, one defines

$$
\nu \in \operatorname{Tan}(\mu, x) \Longleftrightarrow \nu=\lim _{i \rightarrow \infty} c_{i}\left(T_{x, \rho_{i}}\right)_{\#} \mu, \text { for some } \rho_{i} \rightarrow \infty \text { and } c_{i} \rightarrow 0 .
$$

In [Pre87], Preiss showed the useful fact that, for $\mu$-almost every $x$, any tangent measure of a tangent measure of $\mu$ at $x$ is itself a tangent measure of $\mu$ at $x$. In fact,

doi:10.5186/aasfm.2011.3636

2010 Mathematics Subject Classification: 54Exx, 28A75, 14M17, 53C17, 22D05, 26 A16.

Key words: Metric tangents, uniqueness of tangents, iterated tangents, Carnot groups, CarnotCarathéodory distances, biLipschitz homogeneous spaces. 
what he first showed is that, for $\mu$-a.e. $x$ and for every $\nu \in \operatorname{Tan}(\mu, x)$, if $y$ is in the support of $\nu$, then the 'translated measure' $\left(T_{y, 1}\right)_{\#} \nu$ is still in $\operatorname{Tan}(\mu, x)$.

We shall consider tangents in the class of complete doubling metric spaces. Let $(X, d)$ be a metric space whose distance is doubling. Gromov showed that one can consider the tangent spaces at a point $x \in X$ as the limits of sequences of pointed metric spaces $\left(X, \rho_{j} d, x\right)$, with $\rho_{j} \rightarrow \infty$, as $j \rightarrow \infty$. We denote the collection of such spaces as $\operatorname{Tan}(X, x)$.

We shall consider doubling-measured metric spaces, i.e., metric spaces endowed with a doubling measure. As a consequence, the distance itself is doubling. We show that the analogue of Preiss's phenomenon holds:

Theorem 1.1. Let $(X, \mu, d)$ be a doubling-measured metric space. Then the following two properties hold.

(1) For $\mu$-almost every $x \in X$, for all $(Y, y) \in \operatorname{Tan}(X, x)$, and for all $y^{\prime} \in Y$ we have $\left(Y, y^{\prime}\right) \in \operatorname{Tan}(X, x)$.

(2) For $\mu$-almost every $x \in X$, for all $(Y, y) \in \operatorname{Tan}(X, x)$, and for all $y^{\prime} \in Y$ we have $\operatorname{Tan}\left(Y, y^{\prime}\right) \subseteq \operatorname{Tan}(X, x)$.

Notice that as pointed metric spaces $(Y, y)$ and $\left(Y, y^{\prime}\right)$ might be different. A limit space is defined up to isometry. Hence, $(Y, y)$ and $\left(Y, y^{\prime}\right)$ are equal when there exists an isometry $f: Y \rightarrow Y$ with the property that $f(y)=y^{\prime}$. Therefore, if it is the case that there is only one tangent metric space at a point $x$ where the conclusion of the part (1) of Theorem 1.1 holds, then such a metric space $(Y, y)$ in $\operatorname{Tan}(X, x)$ has the property that $(Y, y)$ is isometric to $\left(Y, y^{\prime}\right)$, for all $y^{\prime} \in Y$. In other words, the isometry group of $Y$ acts on $Y$ transitively. In conclusion, uniqueness of tangent spaces leads to isometric homogeneity of such tangents.

In the next theorem we completely characterize the tangents that can appear, if in addition the metric space is geodesic. For more general results see Section 2.

Theorem 1.2. Let $(X, \mu, d)$ be a doubling-measured geodesic metric space. Assume that, for $\mu$-almost every $x \in X$, the set $\operatorname{Tan}(X, x)$ contains only one element. Then, for $\mu$-almost every $x \in X$, the element in $\operatorname{Tan}(X, x)$ is a Carnot group $\mathbf{G}$ endowed with a sub-Finsler left-invariant metric with the first layer of the Lie algebra of $\mathbf{G}$ as horizontal distribution.

Recall that a Carnot group $\mathbf{G}$ of step $s \geq 1$ is a connected, simply-connected Lie group whose Lie algebra $\mathfrak{g}$ admits a step $s$ stratification: this means that we can write

$$
\mathfrak{g}=V_{1} \oplus \cdots \oplus V_{s},
$$

with $\left[V_{j}, V_{1}\right]=V_{j+1}$, for $1 \leq j \leq s$, such that $V_{s} \neq\{0\}$ and $V_{s+1}=\{0\}$. The subspace $V_{1}$ is called the first layer of the Lie algebra $\mathfrak{g}$. A sub-Finsler left-invariant metric with $V_{1}$ as horizontal distribution is defined as follows. One fixes a norm $\|\cdot\|$ on $V_{1}$. The space $V_{1}$ defines a left-invariant sub-bundle $\Delta$ of the tangent bundle of G. The norm $\|\cdot\|$ extends left-invariantly on $\Delta$ as well. The triple $(\mathbf{G}, \Delta,\|\cdot\|)$ is a left-invariant sub-Finsler structure for which the Finsler-Carnot-Carathéodory or sub-Finsler distance $d_{C C}$ is defined as, for any $x, y \in \mathbf{G}$,

$$
d_{C C}(x, y):=\inf \left\{\operatorname{Length}_{\|\cdot\|}(\gamma) \mid \gamma \in C^{\infty}([0,1] ; \mathbf{G}), \gamma(0)=x, \gamma(1)=y, \dot{\gamma} \in \Delta\right\} \text {. }
$$

To conclude the introduction, we would like to mention a similar result of Mattila, which as well was obtained by proving a Preiss's phenomenon for measures on locally 
compact groups with metric dilations. Namely, in [Mat05] it is shown that if a measure on such a group has unique tangents, then its tangents are almost surely Haar measures with respect to some closed dilation-invariant subgroup.

1.1. Other consequences. Given a metric space $(X, d)$, we denote the dilated space by a factor $\rho>0$ by

$$
\rho X:=(X, \rho d) .
$$

Fixed a point $x \in X$, we denote by $\operatorname{Tan}(X, x)$ the space of all Gromov-Hausdorff limits of sequences of pointed metric spaces

$$
\left(\rho_{i} X, x\right) \text {, with } \rho_{i} \rightarrow \infty \text {, as } i \rightarrow \infty \text {. }
$$

In the next section we will recall the definition of Gromov-Hausdorff convergence. However, consider that the elements in $\operatorname{Tan}(X, x)$ are defined up to isometric equivalence.

A measure $\mu$ on a metric space $(X, d)$ is said to be doubling, if there exists a constant $C$ such that, for all $x \in X$ and $r>0$,

$$
0 \neq \mu(B(x, 2 r))<C \mu(B(x, r)) .
$$

Notice that if $\mu$ is a doubling measure, then $d$ is a doubling distance, i.e., there is a constant $N$ such that any ball can be covered by $N$ balls of half the radius. Gromov showed that, whenever $(X, d)$ is a doubling metric space, then, for any $x \in X$, the set $\operatorname{Tan}(X, x)$ is non-empty, see [Gro81, Gro99].

The following theorem is a more detailed version of Theorem 1.2. For its proof we will use the work of Gleason-Montgomery-Zippin [MZ74] and the applications by Berestovskiı̌ [Ber88, Ber89a, Ber89b].

Theorem 1.4. Let $(X, \mu, d)$ be a doubling-measured metric space. Let $\Omega \subseteq X$ be the subset of elements $x \in X$ such that the set $\operatorname{Tan}(X, x)$ contains only one element. Then, for $\mu$-almost every $x \in \Omega$, the element in $\operatorname{Tan}(X, x)$ is an isometrically homogeneous space of the following form. There is a Lie group $G$ and a compact subgroup $H<G$, such that the tangent space at the point $x$ is isometric to the manifold $G / H$ equipped with some $G$-invariant distance function.

If, moreover, the distance $d$ is geodesic, then, for $\mu$-almost every $x \in \Omega$, the element in $\operatorname{Tan}(X, x)$ is a Carnot group $\mathbf{G}$ endowed with a sub-Finsler left-invariant metric with the first layer of the Lie algebra of $\mathbf{G}$ as horizontal distribution.

The following result is an application of the previous techniques (i.e., Theorem 1.1) to the theory of biLipschitz homogeneous spaces. We refer to [LD09, LD08] for more results on these spaces. A metric space $(X, d)$ is said to be locally biLipschitz homogeneous if, for every two points $x_{1}, x_{2} \in X$, there are neighborhoods $U_{1}$ and $U_{2}$ of $x_{1}$ and $x_{2}$ respectively and a biLipschitz homeomorphism $f: U_{1} \rightarrow U_{2}$, such that $f\left(x_{1}\right)=x_{2}$. We call finite-dimensional isometrically-homogeneous space a manifold of the form $G / H$, with $G$ a Lie group and $H$ a compact subgroup $H<G$, endowed with some $G$-invariant distance function.

Theorem 1.5. Let $(X, \mu, d)$ be a doubling-measured metric space. Assume that $(X, d)$ is locally biLipschitz homogeneous. Assume also that there are a point $x_{0} \in X$ and, for some $K>1$, a family of $K$-biLipschitz maps $\mathscr{F}$ such that $\mathscr{F}$ is a group, i.e., $\mathscr{F}$ is closed under composition, and, for all pair of tangents $\left(Z_{1}, z_{1}\right),\left(Z_{2}, z_{2}\right) \in$ $\operatorname{Tan}\left(X, x_{0}\right)$, there is a map $\psi \in \mathscr{F}$ with $\psi:\left(Z_{1}, z_{1}\right) \rightarrow\left(Z_{2}, z_{2}\right)$. Assume that any 
element in $\operatorname{Tan}\left(X, x_{0}\right)$ is locally connected. Then, there is a finite dimensional isometrically homogeneous space $G / H$, such that, for all $x \in X$, each element in $\operatorname{Tan}(X, x)$ is biLipschitz equivalent to $G / H$.

Since the isometries form a group, we immediately have the following result. The local connectedness of tangents is not assumed, since it is a consequence of Proposition 3.7.

Corollary 1.6 (of Theorem 1.5). Let $(X, \mu, d)$ be a doubling-measured metric space. Assume that $(X, d)$ is locally biLipschitz homogeneous and that, for some point $x_{0} \in X$ the collection $\operatorname{Tan}\left(X, x_{0}\right)$ contains only one metric space, up to isometric equivalence. Then, there is a finite dimensional isometrically homogeneous space $G / H$, such that, for all $x \in X$, each element in $\operatorname{Tan}(X, x)$ is biLipschitz equivalent to $G / H$.

Next, one should wonder what are the consequences of having tangents equal to Carnot groups. The answer is definitely not easy at least because there are uncountably many Carnot groups. The case when the tangents are Euclidean is relatively easier. Indeed, David and Toro considered such a case in their study of Reifenberg flat metric spaces, see [DT99]. In the particular case when the metric space is nicely embedded in a Hilbert space, then we observe that the standard 'cone criterion' gives the following easy fact.

Corollary 1.7. Let $X$ be a locally compact subset of a separable Hilbert space $H$. Let $d$ be the distance function on $H$ restricted to $X$. Let $\mu$ be a doubling measure for $(X, d)$. Assume that, at $\mu$-almost every point $x \in X$, the dilated spaces

$$
\rho(X-x)
$$

converge in the Hausdorff sense, as $\rho \rightarrow \infty$. Then $X$ is contained in the union of countably many Lipschitz graphs, up to a set of $\mu$-measure 0.

Question 1.8. Which are the metric spaces that can be isometrically embedded in a separable Hilbert space having the property that, almost everywhere, the Gromov tangents can be calculated as Hausdorff tangents?

\section{Tangents as limit of pointed spaces}

A pointed complete metric space $\left(X_{\infty}, d_{\infty}, x_{\infty}\right)$ is a tangent of a metric space $(X, d)$ at the point $x \in X$, if there are Hausdorff approximations

$$
\left\{\phi_{i}:\left(X_{\infty}, d_{\infty}, x_{\infty}\right) \rightarrow\left(X, d_{i}, x\right)\right\}_{i \in \mathbf{N}}
$$

with $d_{i}=\frac{1}{\lambda_{i}} d$ for some $\lambda_{i} \rightarrow 0$ as $i \rightarrow \infty$. By definition, the fact that the $\phi_{i}$ 's are Hausdorff approximations explicitly means that, for all $R \geq 0$ and all $\delta>0$,

$$
\lim _{i \rightarrow \infty} \sup \left\{\left|d_{i}\left(\phi_{i}(y), \phi_{i}(z)\right)-d_{\infty}(y, z)\right|: y, z \in B\left(x_{\infty}, R\right) \subset X_{\infty}\right\}=0
$$

and

$$
\lim _{i \rightarrow \infty} \sup \left\{d_{i}\left(y, \phi_{i}\left(B\left(x_{\infty}, R+\delta\right)\right)\right): y \in B_{d_{i}}(x, R) \subseteq\left(X, d_{i}\right)\right\}=0 .
$$

The first condition says that

$$
\frac{1}{\lambda_{i}} d\left(\phi_{i}(y), \phi_{i}(z)\right) \rightarrow d_{\infty}(y, z)
$$


uniformly in $y$ and $z$ on bounded sets. The second condition can be written as

$$
\lim _{i \rightarrow \infty} \sup \left\{\frac{1}{\lambda_{i}} d\left(y, \phi_{i}\left(B\left(x_{\infty}, R+\delta\right)\right)\right): y \in B\left(x, \lambda_{i} R\right)\right\}=0 .
$$

Roughly speaking, this means that the sequence of (smaller and smaller) sets $\phi_{i}(B$ $\left.\left(x_{\infty}, R+\delta\right)\right)$ covers $B\left(x, \lambda_{i} R\right)$ better and better, in fact with a sub-linear gap.

\section{Proofs of the results}

Given a Radon measure $\mu$ on a space $X$, one can consider the outer measure $\mu^{*}$, defined for any $A \subseteq X$ by

$$
\mu^{*}(A):=\inf \{\mu(B): B \text { Borel, } B \supseteq A\} .
$$

Even if any geometric intuition says that the following fact is obvious, it is the key point implying Theorem 1.1.

Proposition 3.1. Let $(X, \mu, d)$ be a doubling-measured metric space. Let $A \subseteq$ $X$ be any set and let $a \in A$ be a point of density for $A$, i.e.,

$$
\lim _{r \downarrow 0} \frac{\mu^{*}\left(A \cap B_{r}(a)\right)}{\mu\left(B_{r}(a)\right)}=1 .
$$

Then $\operatorname{Tan}(A, d, a)=\operatorname{Tan}(X, d, a)$.

Corollary 3.2 (of the proof of Proposition 3.1). If $(Y, y) \in \operatorname{Tan}(X, x)$ and $x$ is a point of density for a set $A$, then there are Hausdorff approximations $\phi_{i}: Y \rightarrow X$ such that $\operatorname{Im}\left(\phi_{i}\right) \subseteq A$.

Lemma 3.3. Let $(X, \mu, d)$ be a doubling-measured metric space. Let $A \subseteq X$ be any set and let $a \in A$ be a point of density for $A$. Then, for all $\delta>0$ and all $R \geq 0$, we have

$$
\lim _{\lambda \rightarrow 0} \sup \left\{\frac{1}{\lambda} d(p, A \cap B(a,(R+\delta) \lambda)): p \in B(a, \lambda R)\right\}=0 .
$$

Proof. Fix $\epsilon>0$. Let $C$ and $Q$ be the constants (cf. [Hei01]) of the doubling property for $\mu$, i.e., for all $R>r>0$,

$$
\frac{\mu\left(B_{R}\right)}{\mu\left(B_{r}\right)}<C\left(\frac{R}{r}\right)^{-Q} .
$$

Take $\lambda$ small enough such that

$$
\frac{\mu^{*}(B(a,(R+\delta) \lambda) \backslash A)}{\mu(B(a,(R+\delta) \lambda))}<\alpha:=\frac{1}{2 C}\left(\frac{\epsilon / 2}{2 R+\delta}\right)^{Q} .
$$

We shall prove that, for such a $\lambda$, the supremum is smaller than $\epsilon$. We can assume $\epsilon / 2<\delta$. Assume, by the way of contradiction, that there is some $p \in B(a, \lambda R)$ such that

Note that, by triangle inequality, we have

$$
d(p, A \cap B(a,(R+\delta) \lambda)) \geq \frac{\epsilon}{2} \lambda .
$$

$$
B\left(p, \frac{\epsilon}{2} \lambda\right) \subset B(a,(R+\delta) \lambda)
$$

and thus

$$
A \cap B\left(p, \frac{\epsilon}{2} \lambda\right)=\emptyset
$$


Therefore,

$$
\begin{aligned}
\mu\left(B\left(p, \frac{\epsilon}{2} \lambda\right)\right) & \leq \mu^{*}(B(a,(R+\delta) \lambda) \backslash A) \leq \alpha \mu(B(a,(R+\delta) \lambda)) \\
& \leq \alpha \mu(B(p,(2 R+\delta) \lambda)) \leq \alpha C\left(\frac{\epsilon / 2}{2 R+\delta}\right)^{-Q} \mu\left(B\left(p, \frac{\epsilon}{2} \lambda\right)\right) \\
& \leq \frac{1}{2} \mu\left(B\left(p, \frac{\epsilon}{2} \lambda\right)\right) .
\end{aligned}
$$

This last calculation implies that $1 \leq 1 / 2$, which is a contradiction.

\subsection{Proof of Proposition 3.1.}

Proof of $\operatorname{Tan}(X, a) \subseteq \operatorname{Tan}(A, a)$. Let $\left(X_{\infty}, d_{\infty}, x_{\infty}\right) \in \operatorname{Tan}(X, a)$. So there are Hausdorff approximations

$$
\left\{\phi_{i}:\left(X_{\infty}, d_{\infty}, x_{\infty}\right) \rightarrow\left(X, \frac{1}{\lambda_{i}} d, a\right)\right\}_{i \in \mathbf{N}},
$$

with rescale factors $\lambda_{i} \rightarrow 0$. For all $p \in X_{\infty}$, define $\phi_{i}^{\prime}(p)$ as a closest point in $A$ to $\phi_{i}(p)$. Notice that $A$ might be considered closed, since $A$, the completion $\bar{A}$ of $A$, and the closure $\mathscr{C}(A)$ of $A$ in $X$ have Gromov-Hausdorff distance 0 , therefore they have the same tangents:

$$
\operatorname{Tan}(A, a)=\operatorname{Tan}(\bar{A}, a)=\operatorname{Tan}(\mathscr{C}(A), a) .
$$

So, we constructed maps

$$
\phi_{i}^{\prime}: X_{\infty} \rightarrow A
$$

We claim the following:

Claim 1: $\frac{1}{\lambda_{i}} d\left(\phi_{i}^{\prime}(\cdot), \phi_{i}(\cdot)\right) \rightarrow 0$ uniformly on bounded sets,

Claim 2: The maps

$$
\left\{\phi_{i}^{\prime}:\left(X_{\infty}, d_{\infty}, x_{\infty}\right) \rightarrow\left(A, \frac{1}{\lambda_{i}} d, a\right)\right\}_{i \in \mathbf{N}}
$$

are Hausdorff approximations, and so $\left(X_{\infty}, d_{\infty}, x_{\infty}\right) \in \operatorname{Tan}(A, a)$.

Proof of Claim 1. Fix $R>0$. Observe that, clearly, for any $\delta>0$,

$$
d(\cdot, A) \leq d\left(\cdot, A \cap B\left(a,(R+\delta) \lambda_{i}\right)\right) .
$$

Therefore, Lemma 3.3 gives

$$
\lim _{i \rightarrow \infty} \sup \left\{\frac{1}{\lambda_{i}} d(p, A): p \in B\left(a, \lambda_{i} R\right)\right\}=0 .
$$

Fix some $\eta>0$. For $i$ big enough, we have that, for all $q \in B\left(x_{\infty}, R-\eta\right)$,

$$
\frac{1}{\lambda_{i}} d\left(\phi_{i}(q), a\right) \leq R
$$

By definition of $\phi_{i}^{\prime}$, we have

$$
d\left(\phi_{i}(q), \phi_{i}^{\prime}(q)\right)=d\left(\phi_{i}(q), A\right) .
$$

Thus,

$$
\lim _{i \rightarrow \infty} \sup \left\{\frac{1}{\lambda_{i}} d\left(\phi_{i}(q), \phi_{i}^{\prime}(q)\right): q \in B\left(x_{\infty}, R-\eta\right)\right\}=0
$$


Proof of Claim 2. First,

$$
\begin{aligned}
& \sup \left\{\left|d_{i}\left(\phi_{i}^{\prime}(y), \phi_{i}^{\prime}(z)\right)-d_{\infty}(y, z)\right|: y, z \in B\left(x_{\infty}, R\right) \subset X_{\infty}\right\} \\
& \leq \sup \left\{\left|d_{i}\left(\phi_{i}(y), \phi_{i}(z)\right)-d_{\infty}(y, z)\right|+\left|d_{i}\left(\phi_{i}^{\prime}(y), \phi_{i}(y)\right)\right|+\left|d_{i}\left(\phi_{i}(z), \phi_{i}^{\prime}(z)\right)\right|:\right. \\
& \left.\quad y, z \in B\left(x_{\infty}, R\right)\right\} \\
& \leq \sup \left\{\left|d_{i}\left(\phi_{i}(y), \phi_{i}(z)\right)-d_{\infty}(y, z)\right|: y, z \in B\left(x_{\infty}, R\right)\right\} \\
& \quad+\sup \left\{\left|d_{i}\left(\phi_{i}^{\prime}(y), \phi_{i}(y)\right)\right|: y, z \in B\left(x_{\infty}, R\right)\right\} \\
& \quad+\sup \left\{\left|d_{i}\left(\phi_{i}(z), \phi_{i}^{\prime}(z)\right)\right|: y, z \in B\left(x_{\infty}, R\right)\right\} \\
& \quad \rightarrow 0+0+0=0 .
\end{aligned}
$$

Second,

$$
\begin{aligned}
\sup \left\{d_{i}\left(y, \phi_{i}^{\prime}\left(B\left(x_{\infty}, R+\delta\right)\right)\right): y \in B\left(a, \lambda_{i} R\right) \cap A\right\} \\
\leq \sup \left\{d_{i}\left(y, \phi_{i}^{\prime}\left(B\left(x_{\infty}, R+\delta\right)\right)\right): y \in B\left(a, \lambda_{i} R\right)\right\} \\
\leq \sup \left\{d_{i}\left(y, \phi_{i}\left(B\left(x_{\infty}, R+\delta\right)\right)\right): y \in B\left(a, \lambda_{i} R\right)\right\} \\
\quad+\sup \left\{d_{i}\left(\phi_{i}(z), \phi_{i}^{\prime}\left(B\left(x_{\infty}, R+\delta\right)\right)\right): z \in B\left(a, \lambda_{i} R\right)\right\} \\
\leq \sup \left\{d_{i}\left(y, \phi_{i}\left(B\left(x_{\infty}, R+\delta\right)\right)\right): y \in B\left(a, \lambda_{i} R\right)\right\} \\
\quad+\sup \left\{d_{i}\left(\phi_{i}(z), \phi_{i}^{\prime}(z)\right): z \in B\left(a, \lambda_{i} R\right)\right\} \rightarrow 0 .
\end{aligned}
$$

Proof of $\operatorname{Tan}(A, a) \subseteq \operatorname{Tan}(X, a)$. Vice versa, an element $\left(X_{\infty}, d_{\infty}, x_{\infty}\right) \in \operatorname{Tan}(A, a)$ gives Hausdorff approximations

$$
\left\{\phi_{i}:\left(X_{\infty}, d_{\infty}, x_{\infty}\right) \rightarrow\left(A, \frac{1}{\lambda_{i}} d, a\right)\right\}_{i \in \mathbf{N}},
$$

with rescale factors $\lambda_{i} \rightarrow 0$.

We claim that the following maps are Hausdorff approximations:

$$
\left\{\phi_{i}^{\prime}:\left(X_{\infty}, d_{\infty}, x_{\infty}\right) \rightarrow\left(X, \frac{1}{\lambda_{i}} d, a\right)\right\}_{i \in \mathbf{N}},
$$

defined as

$$
\phi_{i}^{\prime}:=\iota \circ \phi_{i},
$$

where $\iota: A \rightarrow X$ is the inclusion. The first requirement to check is that

$$
d_{i}\left(\phi_{i}^{\prime}(y), \phi_{i}^{\prime}(z)\right)=d_{i}\left(\phi_{i}(y), \phi_{i}(z)\right) \rightarrow d_{\infty}(y, z),
$$

uniformly in $y$ and $z$ on bounded sets, which is clearly true. The second condition is consequence of Lemma 3.3:

$$
\begin{aligned}
& \lim _{i \rightarrow \infty} \sup \left\{d_{i}\left(y, \phi_{i}^{\prime}\left(B\left(x_{\infty}, R+\delta\right)\right)\right): y \in B\left(a, \lambda_{i} R\right)\right\} \\
& \left.\leq \lim _{i \rightarrow \infty} \sup \left\{d_{i}(y, A \cap B(a, R+\delta / 2))\right): y \in B\left(a, \lambda_{i} R\right)\right\} \\
& \quad+\lim _{i \rightarrow \infty} \sup \left\{d_{i}\left(y, \phi_{i}\left(B\left(x_{\infty}, R+\delta\right)\right)\right): y \in B\left(a, \lambda_{i}(R+\delta / 2)\right) \cap A\right\}=0 .
\end{aligned}
$$

Remark 3.4. Both the doubling property and the density point property are necessary in both of the containments in the proof of Proposition 3.1. Indeed, let $(X, d)$ be the Euclidean plane $\mathbf{R}^{2}$. Let $A$ be the subset $\mathbf{R} \times\{0\}$. Notice that $\operatorname{Tan}(X, 0)=\left\{\mathbf{R}^{2}\right\}, \operatorname{Tan}(A, 0)=\{\mathbf{R}\}$, and that no one is contained in the other. Now, if we take $\mu=\mathscr{H}^{2}$, then $\mu$ is doubling, but 0 is not a point of density for $A$. 
On the other hand, if $\mu=\mathscr{H}^{1}\left\llcorner_{A}\right.$, then 0 is a point of density for $A$, but such a $\mu$ is not doubling.

Proof of Corollary 3.2. By Proposition 3.1, the space $(Y, y)$ is in $\operatorname{Tan}(A, x)$. So there are Hausdorff approximations

$$
\psi_{i}: Y \rightarrow A
$$

Consider then the maps $\phi_{i}=\iota \circ \psi_{i}$, where $\iota: A \rightarrow X$ is the inclusion. The calculations at the end of the proof of Proposition 3.1 show that such $\phi_{i}$ 's are Hausdorff approximations for $X$ with image in $A$.

3.2. Some facts on the space of metric spaces. Let $\mathscr{M}$ be a family of separable pointed metric spaces. Assume that $\mathscr{M}$ is 'uniformly totally bounded on bounded sets', namely, for all $R>0$ and for any $\epsilon>0$, there exists a natural number $N=N(\epsilon, R)$ such that every $R$-ball in every $Y \in \mathscr{M}$ admits a covering by $N$ balls of radius $\epsilon$. In particular, a family of uniformly doubling metric space is uniformly totally bounded on bounded sets. In our case, $\mathscr{M}$ will be the collection of the doubling metric space $X$, its dilated $\lambda X$, its tangents, and the iterated tangents. We consider the pointed Gromov-Hausdorff convergence on the set $\mathscr{M}$.

A first fact to recall is that such a topology is metrizable: There exists a distance function $\underline{d}$ on $\mathscr{M}$ such that

$$
\left(X_{\infty}, d_{\infty}, x_{\infty}\right) \in \operatorname{Tan}(X, d, x) \Longleftrightarrow \lim _{\lambda_{i} \rightarrow 0} \underline{d}\left(\left(X, \frac{1}{\lambda_{i}} d, x\right),\left(X_{\infty}, d_{\infty}, x_{\infty}\right)\right)=0 .
$$

A second fact to recall is that the space $(\mathscr{M}, \underline{d})$ is separable. In particular, for each $k \in \mathbf{N}$ there exists a countable cover of sets with diameter less than $1 / 2 k$. Namely,

$$
\mathscr{M}=\bigcup_{l \in \mathbf{N}} B_{l}
$$

such that, if $(Y, y)$ and $\left(Y^{\prime}, y^{\prime}\right)$ are both in $B_{l}$, then

$$
\underline{d}\left((Y, y),\left(Y^{\prime}, y^{\prime}\right)\right)<\frac{1}{2 k} .
$$

\subsection{Proof of Theorem 1.1.}

Proof of Theorem 1.1. We need to show that

$$
\mu\left(\left\{x \in X: \forall(Y, y) \in \operatorname{Tan}(X, x), \forall y^{\prime} \in Y:\left(Y, y^{\prime}\right) \in \operatorname{Tan}(X, x)\right\}^{c}\right)=0 .
$$

In other words,

$$
\mu\left(\left\{x \in X: \exists(Y, y) \in \operatorname{Tan}(X, x), \exists y^{\prime} \in Y:\left(Y, y^{\prime}\right) \notin \operatorname{Tan}(X, x)\right\}\right)=0 .
$$

Using the distance $\underline{d}$ on the collection of metric spaces, we just need to show that, for all $k, m \in \mathbf{N}$, we have

$$
\begin{aligned}
& \mu\left(\left\{x \in X: \exists(Y, y) \in \operatorname{Tan}(X, x), \exists y^{\prime} \in Y: \underline{d}\left(\left(Y, y^{\prime}\right),\left(\frac{1}{t} X, x\right)\right)>\frac{1}{k}, \forall t \in\left(0, \frac{1}{m}\right)\right\}\right) \\
& =0 .
\end{aligned}
$$


Using the cover (3.5) coming from the separability, we need to show that, for all $k, l, m \in \mathbf{N}$, each set

$$
\begin{aligned}
& \left\{x \in X: \exists(Y, y) \in \operatorname{Tan}(X, x), \exists y^{\prime} \in Y:\right. \\
& \left.\quad\left(Y, y^{\prime}\right) \in B_{l} \text { and } \underline{d}\left(\left(Y, y^{\prime}\right),\left(\frac{1}{t} X, x\right)\right)>\frac{1}{k}, \forall t \in\left(0, \frac{1}{m}\right)\right\}
\end{aligned}
$$

is $\mu$-negligible. Assume that one of these sets above is not $\mu$-negligible and call it $A$; so $k, l$ and $m$ are now fixed and $\mu^{*}(A)>0$. Here we use the outer measure $\mu^{*}$, since we don't want, and don't need, to show measurability of such a set.

Let $a$ be a point of density of $A$ for $\mu^{*}$. Since $a \in A$, there exist $(Y, y) \in \operatorname{Tan}(X, a)$ and $y^{\prime} \in Y$ such that $\left(Y, y^{\prime}\right) \in B_{l}$ and $\underline{d}\left(\left(Y, y^{\prime}\right),\left(\frac{1}{t} X, a\right)\right)>\frac{1}{k}$, for all $t \in\left(0, \frac{1}{m}\right)$.

Since $(Y, y) \in \operatorname{Tan}(X, a)$, there is a sequence $\lambda_{i} \rightarrow 0$ such that

$$
\left(\frac{1}{\lambda_{i}} X, a\right) \stackrel{G H}{\rightarrow}(Y, y) .
$$

Let $\phi_{i}: Y \rightarrow X$ the Hausdorff approximations with $\operatorname{Im}\left(\phi_{i}\right) \subseteq A$, given by Corollary 3.2. Let $a_{i}=\phi_{i}\left(y^{\prime}\right) \in A$. Then

$$
\left(\frac{1}{\lambda_{i}} X, a_{i}\right) \stackrel{G H}{\rightarrow}\left(Y, y^{\prime}\right)
$$

Now take $i$ big enough so that

$$
\underline{d}\left(\left(\frac{1}{\lambda_{i}} X, a_{i}\right),\left(Y, y^{\prime}\right)\right)<\frac{1}{2 k} .
$$

Since $a_{i} \in A$, there are spaces $\left(Y_{i}, y_{i}\right) \in \operatorname{Tan}\left(X, a_{i}\right)$ and $y_{i}^{\prime} \in Y_{i}$ such that $\left(Y_{i}, y_{i}^{\prime}\right) \in B_{l}$ and $\underline{d}\left(\left(Y_{i}, y_{i}^{\prime}\right),\left(\frac{1}{t} X, a_{i}\right)\right)>\frac{1}{k}$, for all $t \in\left(0, \frac{1}{m}\right)$. So we arrive at a contradiction:

$$
\begin{aligned}
\frac{1}{k} & <\underline{d}\left(\left(Y_{i}, y_{i}^{\prime}\right),\left(\frac{1}{t} X, a_{i}\right)\right) \leq \underline{d}\left(\left(Y_{i}, y_{i}^{\prime}\right),\left(Y, y^{\prime}\right)\right)+\underline{d}\left(\left(\frac{1}{\lambda_{i}} X, a_{i}\right),\left(Y, y^{\prime}\right)\right) \\
& \leq \operatorname{Diam}_{\underline{d}}\left(B_{l}\right)+\underline{d}\left(\left(\frac{1}{\lambda_{i}} X, a_{i}\right),\left(Y, y^{\prime}\right)\right)<\frac{1}{2 k}+\frac{1}{2 k} .
\end{aligned}
$$

3.4. Proof of Theorem 1.4. Next theorem is well-known in the theory of locally compact groups. It is a consequence of a deep result of Montgomery and Zippin, [MZ74, Corollary on page 243, section 6.3], together with the work [Gle52] of Gleason. An explicit proof can be found in Drutu and Kapovich's lecture notes, [DK11].

Theorem 3.6. (Gleason-Montgomery-Zippin) Let $Y$ be a metric space that is complete, proper, connected, and locally connected. Assume that the isometry group Isom $(Y)$ of $Y$ acts transitively on $Y$. Then Isom $(Y)$ is a Lie group with finitely many connected components.

Proof of Theorem 1.4. Using Theorem 1.1, up to removing a $\mu$-negligible set, we may assume that for all $x \in \Omega$ we have that

$$
\operatorname{Tan}(X, x)=\{(Y, y)\}
$$

and for all $y^{\prime} \in Y$ we also have $\left(Y, y^{\prime}\right) \in \operatorname{Tan}(X, x)$. Thus, $\left(Y, y^{\prime}\right)$ is isometric to $\left(Y, y^{\prime \prime}\right)$ for all $y^{\prime}$ and $y^{\prime \prime} \in Y$. In other words, the metric space $Y$ is isometrically homogeneous. 
Since $Y$ is a tangent of a doubling space, $Y$ is doubling as well. In particular, $Y$ is proper. Moreover, notice that, for all $\lambda>0$, we have $(\lambda Y, y)$ is in $\operatorname{Tan}(X, x)$ and thus it is isometric to $(Y, y)$. Thus, by the next Proposition 3.7, we have that $Y$ is connected and every neighborhood of $y$ contains a connected neighborhood of $y$. By homogeneity we conclude that $Y$ is in fact locally connected.

By Theorem 3.6, the group of isometries $G:=\operatorname{Isom}(Y)$ of $Y$ is a Lie group, and thus $Y$ is homeomorphic to a quotient $G / H$, where $H$ is the stabilizer of a point. Thus there is a $G$-invariant distance on $G / H$ for which $Y$ is isomorphic $G / H$.

If, moreover, $X$ is geodesic, then $Y$ and $G / H$ are geodesic as well. By Berestovskii's Theorem [Ber88], the $G$-invariant distance function on $G / H$ is a $G$-invariant subFinsler metric $d_{S F}$, i.e., there is a $G$-invariant sub-bundle $\Delta$ on the manifold $G / H$ and a $G$-invariant norm on $\Delta$, such that $d_{S F}$ is the Finsler-Carnot-Carathéodory distance associated.

We show now that in fact the space $G / H$ is a Carnot group. Indeed, for all $\lambda>0$, one has $(\lambda Y, y) \in \operatorname{Tan}(X, x)$. Consequently,

$$
\operatorname{Tan}(Y, y) \subseteq \operatorname{Tan}(X, x) .
$$

First, by Mitchell's Theorem [Mit85], the tangent to $Y=G / H$ is a Carnot group G. Second, by uniqueness of tangents, we have that $Y=\mathbf{G}$.

In the above theorem we made used of the following general fact.

Proposition 3.7. Let $\left(Y, y_{0}\right)$ be a proper pointed metric space. Assume that, for all $\lambda>0$, the pointed metric spaces $\left(Y, y_{0}\right)$ and $\left(\lambda Y, y_{0}\right)$ are isometric. Then $Y$ is connected. In fact, any closed metric ball at $y_{0}$ is connected.

Here is a lemma for the proof of the proposition.

Lemma 3.8. In the assumptions of the above proposition, let $\epsilon>0$ and $\bar{y} \in Y$, with $\bar{y} \neq y_{0}$. Then there exists $y^{\prime} \in Y$ such that $d\left(\bar{y}, y^{\prime}\right)<\epsilon$ and $d\left(y^{\prime}, y_{0}\right)<d\left(\bar{y}, y_{0}\right)$.

Proof. For each $\delta \in(0,1]$, consider the set

$$
\Omega_{\delta}:=\left\{f(\bar{y}) \mid f: \delta Y \rightarrow Y \text { isometry with } f\left(y_{0}\right)=y_{0}\right\} .
$$

It is easy to check that $\Omega_{\delta} \subseteq\left\{y \in Y: d\left(y_{0}, y\right)=\delta d\left(y_{0}, \bar{y}\right)\right\}$. The assumption that $\left(\delta Y, y_{0}\right)$ is isometric to $\left(Y, y_{0}\right)$ rephrases as $\Omega_{\delta} \neq \emptyset$. Since the space is proper, we can make use of Ascoli-Arzelà's Theorem. Indeed, for $\delta \in(1 / 2,1)$, pick $z_{\delta} \in \Omega_{\delta}$ and the respective $\delta$-homothety $f_{\delta}$. Using the Ascoli-Arzelà argument to the uniformly Lipschitz maps $f_{\delta}$, we have that there exists a sequence $\delta_{n} \nearrow 1$ for which the maps $f_{\delta_{n}}$ converge uniformly on compact sets to an isometry $f$. In particular, $f\left(y_{0}\right)=y_{0}$ and $f_{\delta_{n}}(\bar{y}) \rightarrow f(\bar{y})$, as $n \rightarrow \infty$.

Set $y_{n}:=f^{-1}\left(f_{\delta_{n}}(\bar{y})\right)$. Then observe that,

$$
d\left(\bar{y}, y_{n}\right)=d\left(f(\bar{y}), f_{\delta_{n}}(\bar{y})\right) \rightarrow 0, \quad \text { as } n \rightarrow \infty,
$$

and

$$
\begin{aligned}
d\left(y_{0}, y_{n}\right) & =d\left(f\left(y_{0}\right), f_{\delta_{n}}(\bar{y})\right)=d\left(y_{0}, f_{\delta_{n}}(\bar{y})\right) \\
& =d\left(f_{\delta_{n}}\left(y_{0}\right), f_{\delta_{n}}(\bar{y})\right)=\delta_{n} d\left(y_{0}, \bar{y}\right)<d\left(y_{0}, \bar{y}\right) .
\end{aligned}
$$

Thus, for $n$ large enough, one can take $y^{\prime}$ as $y_{n}$ for the conclusion of the lemma.

Proof of Proposition 3.7. Assume that a closed ball $\bar{B}\left(y_{0}, R\right)(R>0)$ is not connected. Thus there exist two non-empty closed sets $K_{1}, K_{2} \subseteq \bar{B}\left(y_{0}, R\right)$ such that 
$K_{1} \cap K_{2}=\emptyset$ and $K_{1} \cup K_{2}=\bar{B}\left(y_{0}, R\right)$. Assume $y_{0} \in K_{1}$. Since $Y$ is proper, both $K_{1}$ and $K_{2}$ are compact. Therefore, first $\epsilon:=d\left(K_{1}, K_{2}\right)>0$. Second, there exists $\bar{y} \in K_{2}$ such that $d\left(\bar{y}, y_{0}\right)=d\left(K_{2}, y_{0}\right)$. By the above lemma, there is some $y^{\prime} \in Y$ such that $d\left(\bar{y}, y^{\prime}\right)<\epsilon$ and $d\left(y^{\prime}, y_{0}\right)<d\left(\bar{y}, y_{0}\right)$. By the second inequality, we get that $y^{\prime} \notin K_{2}$ and that $y^{\prime} \in \bar{B}\left(y_{0}, R\right)$. By the first inequality, we get that $y^{\prime} \notin K_{1}$. We just contradicted the fact that $K_{1} \cup K_{2}=\bar{B}\left(y_{0}, R\right)$. Hence $\bar{B}\left(y_{0}, R\right)$ cannot be disconnected.

Finally, since $Y=\bigcup_{R>0} \bar{B}\left(y_{0}, R\right)$, then $Y$ is connected as well.

\subsection{Proof of Theorem 1.5.}

Proof of Theorem 1.5. Let $\Omega \subset X$ be a full-measure set for which the conclusion of Theorem 1.1 holds. Let $x \in \Omega$. Fix any $(Y, y) \in \operatorname{Tan}(X, x)$.

Since $(X, d)$ is biLipschitz homogeneous, there exists an $L$-biLipschitz map

$$
f:\left(U_{x}, x\right) \rightarrow\left(U_{x_{0}}, x_{0}\right),
$$

where $U_{x}$ and $U_{x_{0}}$ are neighborhoods of $x$ and $x_{0}$ respectively. Since $x \in \Omega$, we have, for all $y^{\prime} \in Y$, that $\left(Y, y^{\prime}\right) \in \operatorname{Tan}(X, x)$.

Let $\lambda_{i} \rightarrow 0$ be the rescaling factors giving the tangent $\left(Y, y^{\prime}\right)$. Consider now the same dilations for the set $X$ but now pointed at $x_{0}$ :

$$
\left(X, \frac{1}{\lambda_{i}} d, x_{0}\right)
$$

Up to considering a subsequence, since such dilated spaces are uniformly doubling, the sequence converges to a metric space

$$
\left(Z_{y^{\prime}}, z_{y^{\prime}}\right) \in \operatorname{Tan}\left(X, x_{0}\right) .
$$

Moreover, the $L$-biLipschitz map $f$ induces an $L$-biLipschitz map

$$
f_{y^{\prime}}:\left(Y, y^{\prime}\right) \rightarrow\left(Z_{y^{\prime}}, z_{y^{\prime}}\right) .
$$

Just a remark: as map defined on the set $Y, f_{y^{\prime}}$ could differ from $f_{y^{\prime \prime}}$. The reason is that we are considering metric spaces up to isometric equivalence. We could make explicit the fact that $\left(Y, y^{\prime}\right)$ should be identified via an isometry with another tangent. However, such a rigor will only add heaviness on the reading.

Using the notations of (3.9) and (3.10), we consider the set

$$
G:=\left\{g=f_{y^{\prime \prime}}^{-1} \circ \psi \circ f_{y^{\prime}}: y^{\prime}, y^{\prime \prime} \in Y, \psi:\left(Z_{y^{\prime}}, z_{y^{\prime}}\right) \rightarrow\left(Z_{y^{\prime}}, z_{y^{\prime \prime}}\right), \psi \in \mathscr{F}\right\} .
$$

It is immediate that $G$ is a group of $K L^{2}$-biLipschitz maps which acts transitively on $Y$. By taking the supremum over the $G$-orbit of the distance function, one gets an $K L^{2}$-biLipschitz equivalent metric with respect to which $G$ acts by isometries. Then by Montgomery-Zippin, $G$ is a Lie group. We conclude that any $Y$ is biLipschitz equivalent to $G / H$, where $H$ is the stabilizer of the action. Since the map $f_{y^{\prime}}$ of (3.10) is biLipschitz, then $Z_{y^{\prime}} \in \operatorname{Tan}\left(X, x_{0}\right)$ is biLipschitz equivalent to $G / H$ as well. Since by assumption all tangents at $x_{0}$ are biLipschitz equivalent, then they are all biLipschitz equivalent to the same $G / H$. Finally, by biLipschitz homogeneity, all tangents at any point $x \in X$ are biLipschitz equivalent to the same $G / H$. 


\section{References}

[Ber88] Berestovskĭ̌, V. N.: Homogeneous manifolds with an intrinsic metric. I. - Sibirsk. Mat. Zh. 29:6, 1988, 17-29.

[Ber89a] Berestovskĭ, V. N.: Homogeneous manifolds with an intrinsic metric. II. - Sibirsk. Mat. Zh. 30:2, 1989, 14-28, 225.

[Ber89b] Berestovskĭ̌, V.N.: The structure of locally compact homogeneous spaces with an intrinsic metric. - Sibirsk. Mat. Zh. 30:1, 1989, 23-34.

[DT99] David, G., and T. Toro: Reifenberg flat metric spaces, snowballs, and embeddings. Math. Ann. 315:4, 1999, 641-710.

[DK11] Drutu, C., and M. Kapovich: Lectures on geometric group theory. - Manuscript, 2011.

[Gle52] GLeAson, A. M.: Groups without small subgroups. - Ann. of Math. (2) 56, 1952, 193-212.

[Gro81] Gromov, M.: Groups of polynomial growth and expanding maps. - Inst. Hautes Études Sci. Publ. Math. 53, 1981, 53-73.

[Gro99] Gromov, M.: Metric structures for Riemannian and non-Riemannian spaces. - Progr. Math. 152, Birkhäuser Boston Inc., Boston, MA, 1999.

[Hei01] Heinonen, J.: Lectures on analysis on metric spaces. - Universitext, Springer-Verlag, New York, 2001.

[LD08] Le Donne, E.: Doubling property for biLipschitz homogeneous geodesic surfaces. - J. Geom. Anal. (to appear), doi:10.1007/s12220-010-9167-7.

[LD09] Le Donne, E.: Geodesic manifolds with a transitive subset of smooth biLipschitz maps. - Groups Geom. Dyn. (to appear).

[Mat05] Mattila, P.: Measures with unique tangent measures in metric groups. - Math. Scand. 97:2, 2005, 298-308.

[Mit85] Mitchell, J.: On Carnot-Carathéodory metrics. - J. Differential Geom. 21:1, 1985, 3545.

[MZ74] Montgomery, D., and L. Zippin: Topological transformation groups. - Robert E. Krieger Publishing Co., Huntington, N.Y., 1974 (reprint of the 1955 original).

[Pre87] Preiss, D.: Geometry of measures in $\mathbf{R}^{n}$ : distribution, rectifiability, and densities. - Ann. of Math. (2) 125:3, 1987, 537-643.

Received 28 January 2011 